\title{
DEHN SURGERY AND SATELLITE KNOTS
}

BY

C. MCA. GORDON ${ }^{1}$

\begin{abstract}
For certain kinds of 3-manifolds, the question whether such a manifold can be obtained by nontrivial Dehn surgery on a knot in $S^{3}$ is reduced to the corresponding question for hyperbolic knots. Examples are, whether one can obtain $S^{3}$, a fake $S^{3}$, a fake $S^{3}$ with nonzero Rohlin invariant, $S^{1} \times S^{2}$, a fake $S^{1} \times S^{2}$, $S^{1} \times S^{2} \# M$ with $M$ nonsimply-connected, or a fake lens space.
\end{abstract}

1. Introduction and statement of results. In the present paper we show that for certain kinds of 3-manifolds, the question whether such a manifold can be obtained by nontrivial Dehn surgery on a knot reduces to the corresponding question for simple knots. By Thurston's uniformization theorem for Haken manifolds [27], a knot is simple if and only if it is either hyperbolic (i.e. its complement possesses a complete hyperbolic structure with finite volume), or a torus knot. Since the manifolds obtained by Dehn surgery on the latter can be completely described (see [20], and §7), we essentially have a reduction to the case of hyperbolic knots.

If $K$ is an (unoriented) knot in (oriented) $S^{3}$, and $r=m / n \in \mathbf{Q} \cup\{\infty\},(m, n)=$ 1 , let $(K ; r)$ denote the closed, oriented 3-manifold obtained by Dehn surgery of type $r$ on $K$. (We use the terminology of [21, pp. 258-259]; see \$2.) The unknot is denoted by $O$, so that $(O ; r)$ is the lens space $L(m, n)$, if $|m| \neq 0,1$, or $S^{3}=$ $(O ; 1 / n)$, or $S^{1} \times S^{2}=(O ; 0)$. The manifolds to which our arguments apply are those that are atoroidal, that is, contain no imcompressible torus. Our main result may then be stated as follows. (Throughout, all 3-manifolds are oriented, $\cong$ denotes orientation-preserving homeomorphism, and \# connected sum.)

THEOREM 1.1. Suppose $(K ; r)$ is atoroidal, where $K \neq O$. Then there exist simple knots $K_{0}, K_{1}, \ldots, K_{k}$, with $K_{i} \neq O$ for $1 \leqslant i \leqslant k$, and $K_{0} \neq O$ if $k=0$, together with positive integers $v_{0}=1, v_{1}, \ldots, v_{k}$, such that

$$
(K ; r) \# \underset{i=1}{k}\left(O ; r / v_{i}^{2}\right) \cong \underset{i=0}{k}\left(K_{i} ; r / v_{i}^{2}\right) .
$$

Most of our results are more or less direct consequences of Theorem 1.1.

For instance, in the case of homology spheres, $r=1 / n$, we have the following.

Received by the editors March 6, 1981 and, in revised form, January 25, 1982.

1980 Mathematics Subject Classification. Primary 57M25.

Key words and phrases. Dehn surgery, satellite knots, simple knots.

I Partially supported by NSF Grant MCS78-02995 and an Alfred P. Sloan Research Fellowship. 
THEOREM 1.2. Let $(K ; 1 / n)$ be atoroidal (resp. a homotopy 3-sphere), where $K \neq O$. Then there exist nontrivial simple (resp. hyperbolic) knots $K_{0}, K_{1}, \ldots, K_{k}$ such that

(i) if $n \neq \pm 1$,

$$
(K ; 1 / n) \cong \underset{i=0}{k}\left(K_{i} ; 1 / n\right),
$$

(ii) if $n= \pm 1$,

$$
(K ; \pm 1) \cong \underset{i=0}{j}\left(K_{i} ; \pm 1\right) \# \underset{i=j+1}{\#}\left(K_{i} ; \pm \frac{1}{4}\right),
$$

for some $j,-1 \leqslant j \leqslant k$.

The extra restrictions on the surgery coefficients are consequences of Litherland's results [16] on Dehn surgery on satellite knots with winding number $>1$.

Theorem 1.2 yields the following. (Note that a hyperbolic knot is necessarily nontrivial.)

COROLlARY 1.3. If $S^{3}$ (resp. a fake 3-sphere, resp. a homotopy 3-sphere with nonzero Rohlin invariant) can be obtained as $(K ; 1 / n)$ for some knot $K \neq O$, and $n \neq 0$, then $S^{3}$ (resp. a fake 3-sphere, resp. a homotopy 3-sphere with nonzero Rohlin invariant) can be obtained as $\left(K^{*} ; 1 / n^{*}\right)$ for some hyperbolic knot $K^{*}$, and $n^{*} \neq 0$. Furthermore, $n^{*}=n$, unless, in the first two cases $n= \pm 1$, in which case $n^{*}= \pm 1$ or \pm 4 .

The conjecture that $(K ; 1 / n)$ is never a homotopy 3-sphere for $K \neq O$ and $n \neq 0$, (i.e. that all nontrivial knots have Property $P$ ) has been studied fairly extensively. (See [14, Problem 1.15] and references therein.) Corollary 1.3 shows, in particular, that it is true if and only if it is true for hyperbolic knots.

Closely related to the question whether $(K ; 1 / n)$ can ever be $S^{3}$ for $K \neq O$ and $n \neq 0$ is the question whether knots are determined by their complements. Precisely, we have

THEOREM 1.4. Knots are determined, up to ambient isotopy, by the oriented homeomorphism types of their complements, if and only if hyperbolic knots are.

Next we consider the possibility of obtaining a manifold of the form $S^{1} \times S^{2} \# M$ by Dehn surgery on a knot. Here $M$ is necessarily a homology 3-sphere (possibly $S^{3}$ ), and we must have $r=0$, so that in this case the Dehn surgery is a genuine surgery corresponding to the canonical 0 -framing of $K$. The question whether this can occur for nontrivial $K$ is equivalent to [14, Problem 1.17]. In particular, there is the conjecture that all nontrivial knots have the so-called Property $\mathrm{R}$, i.e. that $(K ; 0)$ cannot be homeomorphic to $S^{1} \times S^{2}$, for $K \neq O$. (See [14, Problem 1.16].)

We shall reduce these questions to the case of hyperbolic knots, but first we have the following relatively straightforward result, which does not use Theorem 1.1.

THEOREM 1.5. If $K$ is a satellite with winding number $>1$, then $(K ; 0)$ is not of the form $S^{1} \times S^{2} \# M$. 
Theorem 1.1 with $r=0$, plus an additional argument, yields

THEOREM 1.6. Suppose $(K ; 0) \cong S^{1} \times S^{2} \# M$, where $K \neq O$. Then there exist hyperbolic knots $K_{0}, K_{1}, \ldots, K_{k}$ such that

$$
\left(K_{i} ; 0\right) \cong S^{1} \times S^{2} \# M_{i}, \quad 0 \leqslant i \leqslant k, \quad \text { and } \quad M \cong \underset{i=0}{k} M_{i} \text {. }
$$

Corollary 1.7. $S^{1} \times S^{2}$ (resp. a fake $S^{1} \times S^{2}$, resp. $S^{1} \times S^{2} \# M$ with $\pi_{1}(M) \neq$ 1) can be obtained as $(K ; 0)$ for some knot $K \neq O$ if and only if $S^{1} \times S^{2}$ (resp. a fake $S^{1} \times S^{2}$, resp. $S^{1} \times S^{2} \# M$ with $\left.\pi_{1}(M) \neq 1\right)$ can be obtained as $\left(K^{*} ; 0\right)$ for some hyperbolic knot $K^{*}$.

That part of Theorem 1.2 which refers to homotopy spheres, Theorem 1.6, and Corollaries 1.3 and 1.7, deal with situations which presumably do not occur. Here is another result of this type. (By a fake lens space we mean a closed, oriented 3-manifold with finite cyclic (possibly trivial) fundamental group, which is not homeomorphic to any lens space, including $S^{3}$.)

THEOREM 1.8. A fake lens space can be obtained as $(K ; r)$ for some knot $K$ if and only if a fake lens space can be obtained as $\left(K^{*} ; r / v^{2}\right)$ for some hyperbolic knot $K^{*}$ and positive integer $v$.

Another consequence of Theorem 1.1 is

THEOREM 1.9. A prime atoroidal manifold $M$ is of the form $(K ; r)$ for some knot $K \neq O$ if and only if it is of the form $\left(K^{*} ; r / v^{2}\right)$ for some simple knot $K^{*}$ and positive integer $v$.

In particular, this applies to Seifert fibre spaces with orbit surface $S^{2}$ and three or fewer singular fibres, for example lens spaces and the Brieskorn homology spheres $\Sigma(p, q, r)$. Again, since it is known exactly which of these occur as the result of Dehn surgery on a torus knot (see [20] and \$7), the question of which others can occur is reduced to the case of hyperbolic knots. (In the case of lens spaces, however, (but only in that case), beware that $K^{*}$ may be trivial.)

Since a hyperbolic 3-manifold is prime and atoroidal, and can never be obtained by Dehn surgery on a torus knot, Theorem 1.9 implies

COROllary 1.10. A hyperbolic 3-manifold $M$ is of the form $(K ; r)$ for some knot $K$ if and only if it is of the form $\left(K^{*} ; r / v^{2}\right)$ for some hyperbolic knot $K^{*}$ and positive integer $v$.

In $[27, \S 5]$, Thurston shows that Dehn surgery on a hyperbolic knot almost always yields a hyperbolic manifold, as a special case of a more general result on attaching solid tori along some of the boundary components of a hyperbolic 3-manifold. Using this more general result (which is also needed in our proof of Theorem 1.1) we prove the following.

THEOREM 1.11. (i) For all $K,(K ; r)$ is irreducible for all but finitely many $r$.

(ii) If $K$ is not a torus knot, then $(K ; r)$ has infinite fundamental group for all but finitely many $r$. 
In particular, this implies Theorem 2 of [17], that all nontrivial knots "almost" have Property $P$.

We remark that (i) is also a special case of a recent result of Hatcher [9].

Our approach is to consider a decomposition of the exterior $X$ of $K$ into atoroidal pieces. By [27], each piece is either hyperbolic or a Seifert fibre space. If the piece containing $\partial X$ is hyperbolic, we apply the general finiteness result of Thurston mentioned above. If it is Seifert fibered, then $K$ is either a composite or a cable knot, and these can be treated directly.

The paper is organized as follows. In $\S 2$ we establish some terminology and conventions. In $\S 3$ we prove some elementary facts about tori in 3-manifolds, and do some homological calculations relating to Dehn surgery on satellite knots. This enables us to prove Theorem 1.5. We also prove the basic reduction lemma (Lemma 3.7) which asserts that if some Dehn surgery on a satellite knot yields an atoroidal manifold, then certain related Dehn surgeries on "simpler" knots yield related atoroidal manifolds. To make this precise, we have to define an appropriate notion of the complexity of a knot. This we do in \$5, after first (in \$4) making some remarks on the decomposition along tori of a knot exterior into atoroidal pieces. The definition of complexity that we use is a combination of the genus of the knot and the maximum number of nonparallel incompressible tori in its exterior. The proof that it has the desired properties uses results of Schubert [22] on the genus of satellite knots and the finiteness theorem of Thurston mentioned above. In $\$ 6$ we prove all the results stated in this introduction, except Theorems 1.5 and 1.11 . In $\$ 7$ we consider the special cases of Dehn surgery on composite and cable knots, the latter providing a good illustration of the reduction process used to prove Theorem 1.1. We examine the case of iterated torus knots in some detail, and in particular describe all the Dehn surgeries on these which yield lens spaces or connected sums of lens spaces. This problem has also been studied by Fintushel-Stern in [3]. However, our point of view provides an alternative to the link calculus approach of [3], and moreover some of the statements in [3] are incorrect. ${ }^{2}$ In $\$ 8$ we give the proof of Theorem 1.11, making use of the results of $\$ 7$. $\$ \$ 9$ and 10 deal with some other aspects of the question whether $(K ; 0)$ can be of the form $S^{1} \times S^{2} \# M$, if $K \neq O$. For instance, in $\$ 9$ we make some remarks on certain group-theoretic questions related to whether this can occur with $\pi_{1}(M) \neq 1$, and in particular observe that this would be impossible if the Whitehead conjecture were true. In $\$ 10$ we show that if $(K ; 0)$ is homeomorphic to $S^{1} \times S^{2} \# M$, then $K$ is homotopy-superslice (see $\S 10$ for definitions).

I should like to thank Ray Lickorish for useful conversations concerning some of this work. I should also like to thank the Departments of Mathematics of the University of California at Santa Barbara and the University of Cambridge for their hospitality during the period in which the work was carried out.

\footnotetext{
${ }^{2}$ See correction to [3], Math. Z. 178 (1981), 143.
} 
2. Terminology. We work in the smooth or $P L$ category.

Let $K$ be an unoriented knot in oriented $S^{3}$. (Two knots are considered to be equal if they are ambient isotopic; similarly for embedded circles in other 3-manifolds.) Let $N(\cdots)$ denote tubular neighbourhood. The exterior of $K$ is $X=S^{3}-$ int $N(K)$. Note that if $K \neq O$, then $\partial X$ is incompressible in $X$.

Let $f: S^{1} \times D^{2} \rightarrow N(K)$ be a homeomorphism which determines the 0 -framing of $K$, i.e. $\left[f\left(S^{1} \times *\right)\right]=0 \in H_{1}(X), * \in \partial D^{2}$. Now choose (arbitrarily) an orientation of $K$. This determines longitude-meridian classes $\lambda, \mu \in H_{1}(\partial X)$ by setting $\lambda=$ $\left[f\left(S^{1} \times *\right)\right], * \in \partial D^{2}$, and $\mu=\left[f\left(* \times \partial D^{2}\right)\right], * \in S^{1}$. If $r=m / n \in \mathbf{Q} \cup\{\infty\}$, $m, n \in \mathbf{Z},(m, n)=1$, then $(K ; r)$, the result of Dehn surgery of type $r$ on $K$, is $X \cup_{h} S^{1} \times D^{2}$, where $h: S^{1} \times \partial D^{2} \rightarrow \partial X$ is a homeomorphism such that $\left[h\left(* \times \partial D^{2}\right)\right]=m \mu+n \lambda \in H_{1}(\partial X), * \in S^{1}$. The manifold $(K ; r)$ is independent of the choice of orientation of $K$ [21, pp. 258-259].

$J$ will always denote a circle in int $S^{1} \times D^{2}$ which does not lie in a ball in $S^{1} \times D^{2}$ and is not a core of $S^{1} \times D^{2}$ (i.e. is not isotopic to $S^{1} \times(0,0)$ ). The winding number of $J$ is the integer $w \geqslant 0$ such that $H_{1}(J) \rightarrow H_{1}\left(S^{1} \times D^{2}\right) \cong \mathbf{Z}$ (unlabelled maps are induced by inclusion) has image $w \mathbf{Z}$. The exterior of $J$ is $Y=S^{1} \times D^{2}-$ int $N(J)$. The hypotheses on $J$ imply that each component of $\partial Y$ is incompressible in $Y$.

Let $K$ be a knot, and $f: S^{1} \times D^{2} \rightarrow N(K)$ as above. Then $J(K)$ denotes the knot $f(J)$ in $S^{3}$. If $K \neq O$, then $J(K)$ is a satellite of $K$, with winding number $w . K$ is simple if it is not a satellite. Recall that a compact 3-manifold $M$ is atoroidal if every incompressible torus in int $M$ is parallel to a component of $\partial M$. Then $K$ is simple if and only if its exterior $X$ is atoroidal.

Let us say that $J$ (as above) is simple if any solid torus $V \subset$ int $S^{1} \times D^{2}$ with $J \subset$ int $V$ has as a core either $J$ or a core of $S^{1} \times D^{2}$. If $Y$ (the exterior of $J$ ) is atoroidal, then $J$ is simple. (One should beware that the converse is false, however. Counterexamples may be obtained by locally tying any prime knot in a core of $S^{1} \times D^{2}$. A less obvious kind of counterexample is illustrated in Figure 1.) It is a consequence of Haken's finiteness theorem (see [10, Lemma 13.2]), applied to incompressible tori, that any satellite knot can be expressed as $J(K)$ with $K \neq O$ and $J$ simple.

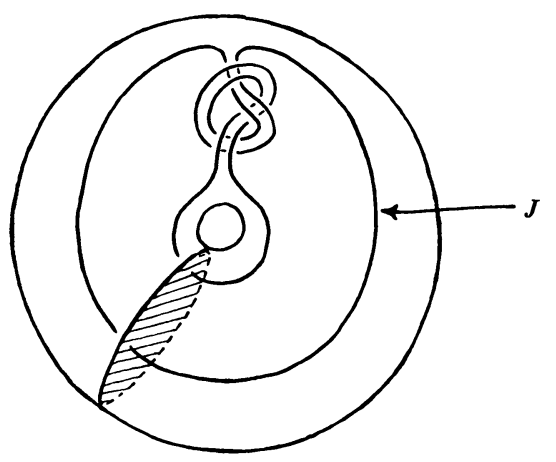

Figure 1 
Let $\alpha, \beta \in H_{1}\left(S^{1} \times \partial D^{2}\right)$ be given by $\alpha=\left[S^{1} \times *\right], * \in \partial D^{2}, \beta \in\left[* \times \partial D^{2}\right]$, $* \in S^{1}$. Note that $H_{1}(Y) \cong \mathbf{Z} \alpha \oplus \mathbf{Z} \mu_{1}$, where $\mu_{1}$ is the class of a meridian of $N(J)$. (We shall sometimes not distinguish notationally between a homology class and its image under a map induced by inclusion.) There exists a homeomorphism $g$ : $S^{1} \times D^{2} \rightarrow N(J)$ which determines the 0 -framing of $J$, i.e. such that, for $* \in \partial D^{2}$, [ $\left.g\left(S^{1} \times *\right)\right]=w \alpha$ in $H_{1}(Y)$. Then $\lambda_{1}=\left[g\left(S^{1} \times *\right)\right] \in H_{1}(\partial N(J))$ is a longitude class of $J$. For $r=m / n \in \mathbf{Q} \cup\{\infty\}$, we define $(J ; r)$ to be $Y \cup_{h} S^{1} \times D^{2}$, where $h$ : $S^{1} \times \partial D^{2} \rightarrow \partial N(J)$ is a homeomorphism such that $\left[h\left(* \times \partial D^{2}\right)\right]=m \mu_{1}+n \lambda_{1} \in$ $H_{1}(\partial N(J))$. Note that for all $K$, if $f, g$ are as above, then $f g$ determines the 0 -framing of $J(K)$. Hence $(J(K) ; r) \cong X \cup(J ; r)$, identified via the restriction of $f$ to $\partial(J ; r)$ $=\partial\left(S^{1} \times D^{2}\right) \rightarrow \partial N(K)=\partial X$.

For $s \in \mathbf{Z}$, let $t_{s}: S^{1} \times D^{2} \rightarrow S^{1} \times D^{2}$ be the homeomorphism given by $t_{s}(\theta,(r, \varphi))=(\theta,(r, \varphi+s \theta))$. If $J \subset$ int $S^{1} \times D^{2}$, let $J_{s}=t_{s}(J)$. Thus $J_{s}$ is the image of $J$ under the operation of cutting $S^{1} \times D^{2}$ along a meridian disc, twisting $s$ times, and regluing.

By a $(p, q)$-curve on the boundary of a framed solid torus $V$ we mean a simple closed curve which represents $p \cdot$ meridian $+q \cdot$ longitude in $H_{1}(\partial V)$. Let $C_{p, q} \subset$ $S^{1} \times D^{2}$ be a $(p, q)$-curve on $S^{1} \times \frac{1}{2} D^{2}$. If $K$ is any knot, $C_{p, q}(K)$ is the $(p, q)$-cable of $K$, and in particular, $C_{p, q}(O)=T_{p, q}$ is the $(p, q)$-torus knot. When we use the notation $C_{p, q}$, we always assume (for nontriviality, and because our knots are unoriented) that $q \geqslant 2$ ( $p$ may be negative).

If $M$ is a closed, (connected) 3-manifold, $M^{-}$will denote $M-$ int $B$, where $B \subset M$ is a 3-ball.

$\mathbf{Z}_{n}$ denotes $\mathbf{Z} / n \mathbf{Z}$.

$\langle\cdots\rangle$ denotes the normal closure of a set of elements in a group.

Throughout, $K$ will denote a knot in $S^{3}, X$ the exterior of $K, J$ a simple closed curve in int $S^{1} \times D^{2}$ (nontrivial in the sense described above), $Y$ the exterior of $J, w$ the winding number of $J$, and $r=m / n \in \mathbf{Q} \cup\{\infty\}$.

3. Preliminary lemmas. We begin with two simple geometric lemmas.

LEMma 3.1. Let $M$ be a closed, orientable 3-manifold, and $T \subset M$ a compressible separating torus. Then $T$ bounds $S^{1} \times D^{2} \# P$ for some summand $P$ of $M$.

Proof. By the loop theorem-Dehn lemma, there exists a 2-disc $D \subset M$ such that $D \cap T=\partial D$ is essential on $T$. Surgery on $T$ using $D$ yields a 2-sphere $S \subset M$. Since $T$ separates $M$ by hypothesis, so does $S$. Thus $M \cong P \# Q$, where the closures of the components of $M-S$ are $P^{-}$and $Q^{-}$. Dually, $T$ is obtained from $S$ by the surgery corresponding to the attachment of a 1-handle $h^{1}$ to (say) $P^{-}$. Then $T$ bounds $P^{-} \cup h^{1} \cong S^{1} \times D^{2} \# P$.

The following lemma can be refined, but will suffice for our purposes.

Lemma 3.2. Let $M_{1}, M_{2}$ be closed, orientable 3-manifolds, and suppose there exists an incompressible torus $T \subset M_{1} \# M_{2}$. Then there exists an incompressible torus $T^{\prime} \subset M_{1}^{-}$or $M_{2}^{-}$, with $\operatorname{im} \pi_{1}\left(T^{\prime}\right)=\operatorname{im} \pi_{1}(T)$ (up to conjugacy). 
Proof. Let $S$ be the 2-sphere which defines the connected sum decomposition $M_{1} \# M_{2}$, and make $T$ transverse to $S$. Then $T \cap S$ consists of a finite collection of disjoint circles; let $C$ be one which is innermost on $S$. Then $C=\partial D$ for some 2-disc $D \subset S$ such that $D \cap T=C$. Also, since $T$ is incompressible, $C=\partial E$ for some 2-disc $E \subset T$. Now $T$ may be modified by replacing $E$ by $D$, and then isotoping $D$ slightly off $S$ in the appropriate normal direction so as to decrease the number of components of $T \cap S$ by at least one. Continuing, this eventually yields a torus $T^{\prime}$ satisfying the conclusion of the lemma.

The next two lemmas are straightforward homological calculations. We use the terminology established in $\$ 2$.

LEMMA 3.3. (i) $H_{1}((J ; r)) \cong \mathbf{Z} \oplus \mathbf{Z}_{(w, m)}$.

(ii) The kernel of $H_{1}(\partial(J ; r)) \rightarrow H_{1}((J ; r))$ is the cyclic subgroup generated by

$$
\begin{cases}\frac{n w^{2}}{(w, m)} \alpha+\frac{m}{(w, m)} \beta, & \text { if } w \neq 0 \\ \beta, & \text { if } w=0 .\end{cases}
$$

Proof. (i) $H_{1}((J ; r)) \cong H_{1}(Y) /\left(n \lambda_{1}+m \mu_{1}\right) \cong\left(\mathbf{Z} \alpha \oplus \mathbf{Z} \mu_{1}\right) /\left(n w \alpha+m \mu_{1}\right)$. Thus $H_{1}((J ; r))$ is presented by the matrix $(n w m)$. By the euclidean algorithm, this is equivalent to $(0 d)$, where $d=(n w, m)=(w, m)($ since $(m, n)=1)$.

(ii) Let $G=\operatorname{ker}\left(H_{1}(\partial(J ; r)) \rightarrow H_{1}((J ; r))\right)$. Since $\beta \mapsto w \mu_{1}$ in $H_{1}(Y)$, we have (see (i) above) that $a \alpha+b \beta \in G$ if and only if, for some $k \in \mathbf{Z}$,

$$
a \alpha+b w \mu_{1}=k\left(n w \alpha+m \mu_{1}\right)
$$

in $H_{1}(Y)=\mathbf{Z} \alpha \oplus \mathbf{Z} \mu_{1}$; that is $a=k n w, b w=k m$.

If $w=0$, then $a=0$, and $b$ is arbitrary, so that $G$ is generated by $\beta$.

If $w \neq 0$, then the least positive $k$ satisfying $b w=k m$ is $w /(w, m)$. The corresponding values of $a$ and $b$ are then $n w^{2} /(w, m)$ and $m /(w, m)$ respectively, giving the stated result.

Recall $(\S 2)$ that $(J(K) ; r) \cong X \cup(J ; r)$.

Lemma 3.4. Let $T \subset(J(K) ; r)$ be the torus along which $X$ and $(J ; r)$ are identified. Then the image of $H_{1}(T) \rightarrow H_{1}((J(K) ; r)) \cong \mathbf{Z}_{m}$ is $w \mathbf{Z}_{m}$.

Proof. Under $H_{1}(T) \rightarrow H_{1}(X) \cong \mathbf{Z}, \alpha \mapsto 0$ and $\beta \mapsto 1$, and $H_{1}((J(K) ; r)) \cong$ $H_{1}((J ; r)) /(\alpha) \cong \mathbf{Z} \mu_{1} /\left(m \mu_{1}\right)$. Since $\beta \mapsto w \mu_{1}$ under $H_{1}(T) \rightarrow H_{1}((J ; r))$, the result follows.

Let $M$ be a closed, orientable 3-manifold with $H_{1}(M) \cong \mathbf{Z}_{m}, m \in \mathbf{Z}$. If $v \in \mathbf{Z}$, let us temporarily say that $M$ is $v$-atoroidal if $M$ contains no incompressible separating torus $T$ such that the image of $H_{1}(T) \rightarrow H_{1}(M)$ is $v \mathbf{Z}_{m}$. (Of course, "separating" is only relevant if $m=0$.)

LEMmA 3.5. If $(J(K) ; r)$ is $w$-atoroidal, where $K \neq O$, then $(J ; r) \cong S^{1} \times D^{2} \# P$ for some summand $P$ of $(J(K) ; r)$. 
Proof. Let $T \subset(J(K) ; r)$ be the torus along which $X$ and $(J ; r)$ are identified. By Lemma 3.4 and the assumption that $(J(K) ; r)$ is $w$-atoroidal, $T$ is compressible. Since $K \neq O, T$ is incompressible in $X$, and the result now follows from Lemma 3.1.

LEMMA 3.6. If $(J(K) ; r)$ is $w$-atoroidal, where $K \neq O$, then either $w=0$ or $(w, m /(w, m))=1$.

Proof. By Lemma $3.5,(J ; r) \cong S^{1} \times D^{2} \# P$. In particular, $\operatorname{ker}\left(H_{1}(\partial(J ; r)) \rightarrow\right.$ $\left.H_{1}((J ; r))\right)$ contains a primitive element. By Lemma 3.3(ii), this implies that either $w=0$ or $\left(n w^{2} /(w, m), m /(w, m)\right)=1$. But

$$
\left(\frac{n w^{2}}{(w, m)}, \frac{m}{(w, m)}\right)=\left(n w, \frac{m}{(w, m)}\right)=\left(w, \frac{m}{(w, m)}\right),
$$

hence the result.

We can now give the

Proof of Theorem 1.5. By Lemma 3.2, $S^{1} \times S^{2} \# M$ ( $M$ a homology sphere) is $v$-atoroidal for all $v \neq 0$. But by Lemma 3.6, $(J(K) ; 0), K \neq O$, can be $w$-atoroidal only if $w=0$ or 1 .

The next lemma is the basis of the reduction procedure which eventually yields Theorem 1.1. We remark that the notion of $v$-atoroidality was introduced essentially only to prove Theorem 1.5 , and will not be used subsequently, although we retain it in the statement of Lemma 3.7.

Lemma 3.7. Suppose $(J(K) ; r)$ is $w$-atoroidal, where $K \neq O$. Then

(i) there exists $P$ such that

$$
(J(K) ; r) \cong\left(K ; r / w^{2}\right) \# P, \text { and }(J(O) ; r) \cong\left(O ; r / w^{2}\right) \# P ;
$$

(ii) if $w=0$, then $(J(K) ; r) \cong\left(J_{s}(O) ; r\right)$ for all $s$.

Proof. By Lemma $3.5,(J ; r) \cong S^{1} \times D^{2} \# P$ for some summand $P$ of $(J(K) ; r)$. Therefore $(J(K) ; r) \cong X \cup\left(S^{1} \times D^{2} \# P\right)$. Now under the identification of $\partial X$ with $\partial(J ; r), \lambda$ corresponds to $\alpha$, and $\mu$ to $\beta$. By Lemma 3.3(ii), $* \times \partial D^{2} \subset$ $\partial\left(S^{1} \times D^{2} \# P\right)=\partial(J ; r)$ represents $n w^{2} \alpha /(w, m)+m \beta /(w, m)$, if $w \neq 0$, or $\beta$, if $w=0$. Hence

$$
(J(K) ; r) \cong\left(X \cup S^{1} \times D^{2}\right) \# P \cong\left(K ; m / n w^{2}\right) \# P=\left(K ; r / w^{2}\right) \# P .
$$

(Note that if $w=0, r / w^{2}$ is to be interpreted as $\infty$, even if $r=0$.)

The same argument, with $O$ in place of $K$ (so that $X$ is replaced by a solid torus), shows that $(J(O) ; r) \cong\left(O ; r / w^{2}\right) \# P$. This proves (i).

To prove (ii), suppose $w=0$. Then $\left(K ; r / w^{2}\right)=(K ; \infty) \cong S^{3}$, and the above argument gives $(J ; r) \cong S^{1} \times D^{2} \#(J(K) ; r)$. Hence, for any $s$,

$$
\left(J_{s}(O) ; r\right) \cong S^{1} \times D^{2} \cup\left(S^{1} \times D^{2} \#(J(K) ; r)\right) \cong(J(K) ; r) .
$$

(The fact that $S^{1} \times D^{2} \cup S^{1} \times D^{2}$ must be $S^{3}$ follows simply by comparing the homology of both sides, although of course it is not hard to work out the explicit boundary identification.) 
The equations in (i) combine to give the symmetric equation

$$
(J(K) ; r) \#\left(O ; r / w^{2}\right) \cong(J(O) ; r) \#\left(K ; r / w^{2}\right),
$$

which is the only form in which Lemma 3.7(i) will be used in the proof of Theorem 1.1 .

The argument used to prove (ii) above generalizes easily to show that, if $P$ is as in (i), then, for all $s$,

$$
\left(J_{s}(O) ; r+s w^{2}\right) \cong\left(O ; r / w^{2}+s\right) \# P .
$$

We make no use of this, however.

4. Atoroidal decompositions of knot exteriors. Throughout this section, we shall restrict to 3-manifolds that are compact, oriented, irreducible, and whose boundary components are incompressible tori.

Let $\mathcal{T}$ be the union of a collection of disjoint, nonparallel, nonperipheral, incompressible tori in the interior of such a 3-manifold $M$, and let $|\mathcal{T}|$ denote the number of components of $\mathcal{T}$. Then by Haken's finiteness theorem [10, Lemma 13.2], the integer $t(M)=\max \{|\sigma|: \mathcal{T}$ as above $\}$ is well defined. $M$ is atoroidal if and only if $t(M)=0$. If $|\mathcal{T}|=t(M)$, say that $\mathcal{T}$ is a maximal system of tori for $M$. Note that if $\mathcal{T}$ is a maximal system, then the components of $M-$ int $N(\mathcal{T})$ are atoroidal. Therefore, by [27], each is either hyperbolic (i.e. its interior has a complete hyperbolic structure with finite volume) or belongs to a small class of Seifert fibre spaces. If they are all of the latter kind, then we shall say that $M$ is a special graphmanifold (see [28]). Thus the special graphmanifolds are precisely those manifolds in the class described at the beginning of this section whose volume (in the sense of Gromov, see $[27, \S 5]$ ) is zero. (We remark that although it turns out that the subsystem of $\mathcal{T}$, obtained by amalgamating as many of the Seifert fibred pieces as possible into larger Seifert fibred pieces, is unique [11, 12], it is only the existence of a maximal system that we shall need.)

Suppose $M=M_{1} \cup M_{2}$, with $M_{1} \cap M_{2}$ a union $\mathcal{T}$ of tori in $\partial M_{i}, i=1,2$, and suppose that no component of $M_{i}$ is homeomorphic to $S^{1} \times S^{1} \times I, i=1,2$. Then clearly

$$
t(M) \geqslant t\left(M_{1}\right)+t\left(M_{2}\right)+|\sigma| .
$$

Now say that $M$ is anannular if every properly embedded annulus in $M$ is parallel into $\partial M$. (It is not hard to show that an atoroidal manifold is either anannular or belongs to a rather small class of Seifert fibred spaces.)

Let $M=M_{1} \cup M_{2}$ as above, and suppose that $M_{1}$ is anannular. Then (*) is actually an equality, since any incompressible torus $T$ in int $M$ can be isotoped off $\mathcal{T}$. (First make $T$ transverse to $\mathcal{T}$. Using a standard innermost disc argument, we may, by an isotopy of $T$, eliminate from $T \cap \mathcal{T}$ all simple closed curves which bound discs on $T$. Thus we may assume that $T \cap \mathcal{T}$ consists of a finite number of parallel essential simple closed curves on $T$, separating $T$ into annuli. If $T \cap \mathcal{T} \neq \varnothing$, at least one of these annuli, $A$, say, is contained in $M_{1}$. Since $M_{1}$ is anannular, $A$ is isotopic (rel $\partial A$ ) into $\mathcal{T}$, and so there is an isotopy of $T$ which eliminates at least $\partial A$ from $T \cap \sigma^{\text {.) }}$ 
Now let $K$ be a knot with exterior $X$, and let $\mathcal{T}$ be a maximal system of tori for $X$. Let $M$ be the component of $X-$ int $N(\tau)$ which contains $\partial X$. The special role played by cable and composite knots in the present context is described in the following lemma.

LEMMA 4.1. If $M$ is a Seifert fibre space, then $K$ is either a torus knot, a cable of a nontrivial knot, or a composite knot (according as $\partial M$ has one, two, or three components).

Proof. It is shown in [11, Lemma VI.3.4] that any Seifert fibre space contained with incompressible boundary in a knot exterior is either the exterior of a torus knot, the exterior of some $C_{p, q}$ in $S^{1} \times D^{2}$, or a (disc-with-holes) $\times S^{1}$. (In the present setting, the only possibility in the latter case is a (disc-with-2-holes) $\times S^{1}$.) It is then not hard to show that these possibilities correspond to the possibilities for $K$ listed in the lemma. We omit the details.

If we define the volume of $K$ to be the volume of $X$ (see [27, §6]), then Lemma 4.1, together with induction on $t(K)$ (see Lemma 5.2), gives

Corollary 4.2. The volume of $K$ is zero if and only if $K$ is in the class generated by $O$ under the operations of connected sum and cabling.

Another description of the above class is that it is the class of fibred knots whose monodromy has all its irreducible components (in the sense of Thurston [26]) of finite order. By [19], it may also be characterized as the class of knots which are attracting closed orbits for a nonsingular Morse-Smale flow on $S^{3}$ (although there the possibility of connected sum seems to have been overlooked).

5. A notion of complexity. Let $g(K)$ denote the genus of $K$.

Lemma 5.1. Suppose $K \neq O$. Then

(i) if $w \geqslant 1, g(K) \leqslant g(J(K))$, and $g(J(O))<g(J(K))$;

(ii) if $w=0, g\left(J_{s}(O)\right) \leqslant g(J(K))$ for all $s$.

Proof. (i) This follows immediately from the inequality

$$
g(J(K)) \geqslant w g(K)=g(J(O)),
$$

which is proved by Schubert in [22, $\$ 12$, p. 192].

(ii) If $w=0$, let $g(J)$ be the minimal genus of an orientable surface spanned by $J$ in $S^{1} \times D^{2}$. Then it follows from [22, §12, Hilfssatz 1] that $g(J(K))=g(J)$. But clearly $g\left(J_{s}(O)\right) \leqslant g(J)$ for all $s$, hence the result.

If $K$ is a nontrivial knot, with exterior $X$, we define $t(K)=t(X)$.

The following is an immediate consequence of (*) of $\$ 4$, with $M_{1}=X, M_{2}=Y$, and $\mathfrak{T}=\partial X$.

Lemma 5.2. If $K \neq O$, then $t(K)<t(J(K))$.

Recall the definition of a simple curve $J \subset$ int $S^{1} \times D^{2}(\S 2)$.

Lemma 5.3. Let $J$ be simple, with $w=0$, and suppose $K \neq O$. Then there exists $s$ such that $J_{s}(O) \neq O$ and $t\left(J_{s}(O)\right)<t(J(K))$. 
Proof. Recall that $Y$ is the exterior of $J$ in $S^{1} \times D^{2}$. The two components of $\partial Y$ are $\partial_{0} Y=\partial\left(S^{1} \times D^{2}\right)$, and $\partial_{1} Y=\partial N(J)$. Let $\sigma$ be a maximal system of tori for $Y$. We claim that $\partial_{0} Y$ and $\partial_{1} Y$ lie in the same component $M$ of $Y-$ int $N(\mathcal{T})$, and that $M$ is not a Seifert fibred space.

To establish the first assertion, let $T$ be any incompressible torus in $Y$. By an argument like that used in the proof of Lemma 3.1, $T$ either bounds a solid torus $V$ in $S^{1} \times D^{2}$, or lies in a ball in $S^{1} \times D^{2}$. If the former holds, we must have $J \subset V$ since $T$ is incompressible in $Y$. Since $J$ is simple, $T$ must then be parallel to either $\partial_{0} Y$ or $\partial_{1} Y$.

Hence each component $T$ of $\mathcal{T}$ lies in a ball in $S^{1} \times D^{2}$, and therefore $\partial_{0} Y$ and $\partial_{1} Y$ can be joined by a path in $Y$ missing $T$. It follows that they can be joined by a path in $Y$ missing $\mathcal{T}$, that is, $\partial_{0} Y$ and $\partial_{1} Y$ belong to the same component of $Y-$ int $N(\mathcal{T})$.

We now show that $M$ is not Seifert fibred. This follows from considerations like those in [11, Lemma VI.3.4] (see Lemma 4.1 above), but for completeness we include a proof anyway.

If $M$ were Seifert fibred, then there would exist an annulus $A \subset M$, namely the $S^{1}$-bundle over an arc in the orbit surface joining the images of $\partial_{0} Y$ and $\partial_{1} Y$, and missing the images of the singular fibres, with one component of $\partial A$ in $\partial_{0} Y$ and the other in $\partial_{1} Y$. Now consider the standard (unknotted, 0 -framed) embedding of $S^{1} \times D^{2}$ in $S^{3}$, and let $C$ be the core of the complementary solid torus. In this way regard $Y$ as the exterior of the 2-component link $C \cup J$. Let $\lambda_{i}, \mu_{i} \in H_{1}\left(\partial_{i} Y\right)$, $i=0,1$, be longitude-meridian pairs for $C, J$ respectively. Note that, since the linking number $\operatorname{Lk}(C, J)=w=0, \lambda_{i} \mapsto 0$ in $H_{1}(Y) \cong \mathbf{Z} \mu_{0} \oplus \mathbf{Z} \mu_{1}, i=0,1$. Since $\partial A \cap \partial_{i} Y, i=0,1$, are homologous in $Y$, we must then have $\left[\partial A \cap \partial_{i} Y\right]= \pm \lambda_{i} \in$ $H_{1}\left(\partial_{i} Y\right), i=0,1$. Therefore $A$ extends to an annulus $A^{\prime} \subset S^{3}$ with $\partial A^{\prime}=C \cup J$. But $C$ is unknotted, and $\operatorname{Lk}(C, J)=0$, so that $C \cup J$ is the unlink, contradicting the assumption that $J$ does not lie in a ball in $S^{1} \times D^{2}$.

The exterior of $J(O)$ is homeomorphic to $S^{1} \times D^{2} \cup Y$, attached via some homeomorphism $h: S^{1} \times \partial D^{2} \rightarrow \partial_{0} Y$. The exterior of $J_{s}(O)$ is then $S^{1} \times D^{2} \cup Y$, attached via $h g_{s}$, where $g_{s}: S^{1} \times \partial D^{2} \rightarrow S^{1} \times \partial D^{2}$ is given by $g_{s}(\theta, \varphi)=$ $(\theta+s \varphi, \varphi)$. We regard this as $\left(S^{1} \times D^{2} \cup M\right) \cup(\overline{Y-M})=M_{s} \cup(\overline{Y-M})$, say. Now by [27, Theorem 5.9], for all but finitely many integers $s, M_{s}$ is hyperbolic, and is therefore irreducible, atoroidal, anannular, and has incompressible boundary. Choose such an $s$. Then $J_{s}(O) \neq O$, since $\partial_{1} Y$ is incompressible in $M_{s} \cup(\overline{Y-M})$. Also, from (*) of $\S 4$ (with equality) we have

$$
t\left(J_{s}(O)\right)=t(\overline{Y-M})+|\widetilde{\tau}| .
$$

On the other hand, the exterior of $J(K)=X \cup Y=X \cup M \cup(\overline{Y-M})$, so that (if $K \neq O)(*)$ gives

$$
t(J(K)) \geqslant t(X)+t(\overline{Y-M})+|\mathcal{T}|+1>t\left(J_{s}(O)\right),
$$

as required.

We now define the complexity $c(K)$ of a knot $K$ by $c(K)=(g(K), t(K))$, and order lexicographically, so that $c\left(K_{1}\right)<c\left(K_{2}\right)$ if and only if either $g\left(K_{1}\right)<g\left(K_{2}\right)$, or $g\left(K_{1}\right)=g\left(K_{2}\right)$ and $t\left(K_{1}\right)<t\left(K_{2}\right)$. (We also set $t(O)=0$.) 
The following is an immediate consequence of Lemmas 5.1, 5.2, and 5.3.

LEMMA 5.4. Suppose $K \neq O$. Then

(i) if $w \geqslant 1, c(K)<c(J(K))$, and $c(J(O))<c(J(K))$;

(ii) if $w=0$ and $J$ is simple, there exists such that $J_{s}(O) \neq O$ and $c\left(J_{s}(O)\right)<$ $c(J(K))$.

\section{Main proofs.}

Proof of THEOREM 1.1. Since the set of ordered pairs of nonnegative integers is well ordered by the lexicographic ordering, we may prove this by induction on $c(K)$.

So suppose $(K ; r)$ is atoroidal, $K \neq O$.

If $K$ is simple, we take $k=0, K_{0}=K$.

If $K$ is not simple, then $K=J\left(K^{\prime}\right)$, say, where $K^{\prime} \neq O$ and $J$ is simple. There are now two cases.

(1) $w=0$. By Lemma 5.4(ii), there exists $s$ such that $J_{s}(O) \neq O$ and $c\left(J_{s}(O)\right)<$ $c(K)$. By Lemma $3.7(\mathrm{ii}),\left(J_{s}(O) ; r\right) \cong(K ; r)$. Hence the result follows by induction.

(2) $w \geqslant 1$. By Lemma 3.7(i) we have

$$
(K ; r) \#\left(O ; r / w^{2}\right) \cong(J(O) ; r) \#\left(K^{\prime} ; r / w^{2}\right) .
$$

In particular, $(J(O) ; r)$ and $\left(K^{\prime} ; r / w^{2}\right)$ are atoroidal. Furthermore, by Lemma 5.4(i), $c\left(K^{\prime}\right)<c(K)$, and $c(J(O))<c(K)$. Therefore, by inductive hypothesis,

$$
\left(K^{\prime} ; r / w^{2}\right) \# \underset{i=1}{k^{\prime}}\left(O ; r / w^{2} v_{i}^{\prime 2}\right) \cong \prod_{i=0}^{k^{\prime}}\left(K_{i}^{\prime} ; r / w^{2} v_{i}^{\prime 2}\right),
$$

as in the statement of the theorem. Similarly, if $J(O) \neq O$,

$$
(J(O) ; r) \# \underset{i=1}{k^{*}}\left(O ; r / v_{i}^{* 2}\right) \cong \underset{i=0}{k^{*}}\left(K_{i}^{*} ; r / v_{i}^{* 2}\right),
$$

say. If $J(O)=O$, we still have a (trivial) equation of this form, by taking $k^{*}=0$, $K_{0}^{*}=O, v_{0}^{*}=1$. In either case, (a), (b), and (c) yield

$$
\begin{aligned}
(K ; r) \#\left(O ; r / w^{2}\right) \# \underset{i=1}{\#}\left(O ; r / v_{i}^{* 2}\right) \underset{i=1}{k^{*}}\left(O ; r / w^{2} v_{i}^{\prime 2}\right) \\
\\
\cong \underset{i=0}{\#}\left(K_{i}^{*} ; r / v_{i}^{* 2}\right) \underset{i=0}{k^{*}}\left(K_{i}^{\prime} ; r / w^{2} v_{i}^{\prime 2}\right) .
\end{aligned}
$$

If $K_{0}^{\prime} \neq O$, this is an equation of the desired form for $(K ; r)$. If $K_{0}^{\prime}=O$, the desired equation is obtained by cancelling $\left(O ; r / w^{2}\right)$ on the left-hand side with $\left(K_{0}^{\prime} ; r / w^{2} v_{0}^{\prime 2}\right)=\left(O ; r / w^{2}\right)$ on the right.

Proof of Theorem 1.2. Without the extra restrictions on the surgery coefficients, the result is an immediate consequence of Theorem 1.1, and (in the homotopy sphere case) the fact that torus knots have Property $P$.

The restrictions on the surgery coefficients follow from a result of Litherland [16]. Although not explicitly stated, the proof in [16] (see particularly Proposition 2 and its proof, and the proof of Theorem 1 in $\S 7)$ shows that if $(J ; 1 / n) \cong S^{1} \times D^{2} \# P$, where $n \neq 0$, then either $w=0$ or 1 , or $w=2$ and $n= \pm 1$. (In fact, it is shown that in the latter case, only one of the values \pm 1 is possible, but we shall not use this.) 
Now recall that in the inductive step in the proof of Theorem 1.1, consideration of $(K ; 1 / n)$ is replaced by consideration of either $\left(J_{s}(O) ; 1 / n\right)$ (if $\left.w=0\right)$, or $(J(O) ; 1 / n)$ and $\left(K^{\prime} ; 1 / n w^{2}\right)$ (if $\left.w \geqslant 1\right)$. Therefore, if $|n|>1$, so that $w=0$ or 1 , the surgery coefficient remains unchanged, whereas if $n= \pm 1$, so that $w=0,1$, or 2 , the surgery coefficient either remains unchanged or becomes $\pm \frac{1}{4}$. This implies the stated result.

Proof of Corollary 1.3. This is an immediate consequence of Theorem 1.2, and the fact that the Rohlin invariant of $\left(K ; \pm \frac{1}{4}\right)$ is necessarily 0 . (The Rohlin invariant of $(K ; 1 / n)$ is $n \alpha(K)$, where $\alpha(K)$ is the arf invariant of $K$ [5].)

Proof of TheOrem 1.4. This will follow at once from Theorem 1.1 once we have established the following claim: If $K \neq O$, then there exist $K^{\prime} \neq K$ and an orientation-preserving homeomorphism $X^{\prime} \rightarrow X$ if and only if $(K ; 1 / n) \cong S^{3}$ for some $n \neq 0$.

To do this, first suppose we have $K^{\prime} \neq K$ and an orientation-preserving homeomorphism $g: X^{\prime} \rightarrow X$. Let $\lambda, \mu$ be a longitude-meridian pair determined by some orientation of $K$. Then, for some orientation of $K^{\prime}$, and corresponding longitudemeridian pair $\lambda^{\prime}, \mu^{\prime}$, we have $\left(g \mid \partial X^{\prime}\right)_{*}\left(\lambda^{\prime}\right)=\lambda,\left(g \mid \partial X^{\prime}\right)_{*}\left(\mu^{\prime}\right)=\mu+n \lambda$, for some $n \in \mathbf{Z}$. Hence $(K ; 1 / n) \cong\left(K^{\prime} ; \infty\right) \cong S^{3}$. Also, if $n=0$ then $g$ would extend to an orientation-preserving homeomorphism $\left(S^{3}, K^{\prime}\right) \rightarrow\left(S^{3}, K\right)$, implying that $K^{\prime}=K$.

Conversely, suppose $(K ; 1 / n) \cong S^{3}$, for some $n \neq 0$. So $S^{3} \cong X \cup_{h} S^{1} \times D^{2}$, where $\left[h\left(* \times \partial D^{2}\right)\right]=\mu+n \lambda \in H_{1}(\partial X)$. Let $K^{\prime}$ be the knot represented by the core of $S^{1} \times D^{2}$. If $K=K^{\prime}$, there would exist an orientation-preserving homeomorphism of $S^{3}$ taking $K$ to $K^{\prime}$. This would induce an orientation-preserving homeomorphism $g: X \rightarrow X$ such that $(g \mid \partial X)_{*}(\lambda)=\varepsilon \lambda,(g \mid \partial X)_{*}(\mu)=\varepsilon \mu+n \varepsilon \lambda$, where $\varepsilon=$ \pm 1 . Thus $(g \mid \partial X)_{*}$ would have infinite order, contradicting Johannson's finiteness theorem [12, §27]. (See the discussion in [25, p. 35].)

To apply Theorem 1.1 to the possibility of obtaining $S^{1} \times S^{2} \# M$ by surgery on a nontrivial knot, we need the following lemma.

Lemma 6.1. Suppose $(K ; 0) \cong S^{1} \times S^{2} \# M$. Then $(K ; 0)$ is atoroidal.

Proof. Since any knot group has weight $1, \pi_{1}((K ; r))$ has weight 1 , for all $K, r$. Hence, under the hypothesis of the lemma, $\mathbf{Z} * \pi_{1}(M)$ would have weight 1 .

Now let $M \cong \#_{i=1}^{q} M_{i}$ be a prime factorization of $M$; in particular, each $M_{i}$ is irreducible. If $S^{1} \times S^{2} \# M$ contained an incompressible torus, then, by Lemma 3.2, so would $M_{p}$ for some $p, 1 \leqslant p \leqslant q$. This $M_{p}$ would then be a Haken manifold, and therefore $\pi_{1}\left(M_{p}\right)$ would be residually finite (Thurston, unpublished, see [27]), and nontrivial. But $\mathbf{Z} * \pi_{1}\left(M_{p}\right)$, being a quotient of $\mathbf{Z} * \pi_{1}(M)$, would also have weight 1 , contradicting [4]. (For further discussion of these matters, see $\$ 9$.)

Proof of TheOREM 1.6. In view of Lemma 6.1, this follows from Theorem 1.1 and the fact that for a nontrivial torus knot, $(K ; 0)$ is a bundle over $S^{1}$ with fibre a surface of genus $>0$. (If $K_{0}=O$ in Theorem 1.1, just cancel an $S^{1} \times S^{2}$ on both sides.)

Proof of TheOrem 1.8. First note that since Dehn surgery on a torus knot never yields a fake lens space, it suffices to prove the statement with "hyperbolic" replaced by "simple". 
So suppose $(K ; r)=M$ is a fake lens space. By Theorem 1.1 , there exist simple knots $K_{0}, K_{1}, \ldots, K_{k}$ and positive integers $v_{0}=1, v_{1}, \ldots, v_{k}$ such that, writing $L_{i}=\left(O ; r / v_{i}^{2}\right)$ and $Q_{i}=\left(K_{i} ; r / v_{i}^{2}\right)$, we have

$$
M \# \underset{i=1}{\#} L_{i} \cong \underset{i=0}{k} Q_{i} \text {. }
$$

If $m=1$, the result is contained in Corollary 1.3, so suppose otherwise. Then note that if some $Q_{i} \cong S^{3}$, we must have $i \geqslant 1$ and $L_{i} \cong S^{3}$, so that $M$ satisfies an equation of the form (d) with a smaller value of $k$. We may therefore suppose that no $Q_{i}$ is homeomorphic to $S^{3}$. In that case, if $\pi_{1}\left(Q_{i}\right)=1$ for some $i$, then $Q_{i}$ is a fake 3-sphere, and we are done. If not, then since $\pi_{1}\left(M \# \#_{i=1}^{k} L_{i}\right)$ is a free product of $k+1$ cyclic groups, $\pi_{1}\left(Q_{i}\right)$ must be cyclic, for each $i=0,1, \ldots, k$. But by hypothesis, the left-hand side of (d) is not a connected sum of lens spaces, so some $Q_{i}$ is a fake lens space.

Proof OF Theorem 1.9. By Theorem 1.1,

$$
M \# \underset{i=1}{k}\left(O ; r / v_{i}^{2}\right) \cong \underset{i=0}{k}\left(K_{i} ; r / v_{i}^{2}\right),
$$

with $K_{i}$ simple. Since $M$ is prime, it follows that $\left(K_{j} ; r / v_{j}^{2}\right) \cong M \# \#_{i \in I}\left(O ; r / v_{i}^{2}\right)$ for some $j, 0 \leqslant j \leqslant k$, and some $I \subset\{1, \ldots, k\}$. Let $r / v_{i}^{2}$ expressed in its lowest terms be $m_{i} / n_{i}$. Note that $m_{i} \mid m$. Then

$$
H_{1}\left(M \# \underset{i \in I}{\#}\left(O ; r / v_{i}^{2}\right)\right) \cong \mathbf{Z}_{m} \oplus \bigoplus_{i \in I} \mathbf{Z}_{m_{i}} .
$$

But $H_{1}\left(\left(K_{j} ; r / v_{j}^{2}\right)\right)$ is cyclic, so we must have $\left|m_{i}\right|=1$, and therefore $\left(O ; r / v_{i}^{2}\right) \cong S^{3}$, for all $i \in I$. Therefore $\left(K_{j} ; r / v_{j}^{2}\right)=M$.

7. Composites, cables, and iterated torus knots. As we remarked in $\S 4$, composite and cable knots arise naturally as special cases when one considers a decomposition of the exterior of a knot along tori into atoroidal pieces. We need to examine the effect of Dehn surgery in these cases in order to prove Theorem 1.11.

For composite knots, we have the following. (See also [1, Lemma 2 and 5, §7].)

LEMMA 7.1. If $K$ is composite, then $(K ; r)$ is irreducible and contains an incompressible torus for all $r \in \mathbf{Q}$.

Proof. Suppose $K=K_{1} \# K_{2}$. Then $X \cong X_{1} \cup_{A} X_{2}$, where $X_{i}$ is the exterior of $K_{i}$, and $A \subset \partial X_{i}$ is a meridional annulus, $i=1,2$. Let $r=m / n$ as usual. Then $(K ; r) \cong\left(X_{1} \cup_{A} X_{2}\right) \cup V$, where $V$ is a solid torus. There are longitude-meridian pairs $\lambda, \mu \in H_{1}(\partial X)$ and $\lambda_{0}, \mu_{0} \in H_{1}(\partial V)$ such that the homeomorphism $f: \partial V \rightarrow \partial X$ by means of which $V$ is attached to $X$ satisfies $f_{*}\left(\mu_{0}\right)=m \mu+n \lambda, f_{*}\left(\lambda_{0}\right)=u \mu+v \lambda$, where $m v-n u=1$. Therefore $f_{*}^{-1}(\mu)=v \mu_{0}-n \lambda_{0}$. Hence $(K ; r) \cong\left(X_{1} \cup_{B} V\right) \cup$ $X_{2}$, where $B$ is the annulus $\partial X_{1}-$ int $A$ on $\partial X_{1}$ and a neighbourhood of a $(v,-n)$-curve on $\partial V$. Now since $r \in \mathbf{Q}, n \neq 0$, and so $B$ is incompressible in $V$. Also, $B$ is incompressible in $X_{1}$, and, if $K_{1} \neq O, \partial X_{1}$ is incompressible in $X_{1}$. Hence $W=$ $X_{1} \cup_{B} V$ is irreducible and has incompressible boundary. Thus, if $K_{2} \neq O,(K ; r) \cong$ $W \cup X_{2}$ is irreducible and contains an incompressible torus. 
If $n=1$, so that we have $\left(K_{1} \# K_{2} ; m\right)$, the result of genuine $m$-framed surgery on $K_{1} \# K_{2}$, then, in the above proof, $v=0$ and $B$ is a longitudinal annulus on $\partial V$. Thus $W \cong X_{1}$, and $\left(K_{1} \# K_{2} ; m\right) \cong X_{1} \cup X_{2}$ is obtained by gluing together the exteriors of $K_{1}$ and $K_{2}$. (This has also been observed by S. Akbulut.) In fact, orienting our knots and using the notation of [6] (in that notation, $(K ; m)=$ $M(O, K ; 1,0, m,-1) \cong M(O, K ;-1,0,-m, 1))$, explicitly computing the identification of the boundaries of $X_{1}$ and $X_{2}$ shows that

$$
M\left(O, K_{1} \# K_{2} ;-1,0, m, 1\right) \cong M\left(K_{1}, K_{2} ;-1,0, m, 1\right) .
$$

Next we consider Dehn surgery on cable knots. This provides a good illustration of Lemma 3.7. Recall that we assume $q \geqslant 2$.

LEMMA 7.2.

$$
\left(C_{p, q} ; r\right) \cong \begin{cases}S^{1} \times D^{2} \# L(q, p), & \text { if } r=p q, \\ S^{1} \times D^{2}, & \text { if } m=n p q \pm 1,\end{cases}
$$

and otherwise is a Seifert fibre space with incompressible boundary.

Proof. $S^{1} \times D^{2}$ has a Seifert fibration with orbit surface $D^{2}$ and one singular fibre of multiplicity $q$, in which $C_{p, q}$ is an ordinary fibre. Hence $Y$, the exterior of $C_{p, q}$ in $S^{1} \times D^{2}$, has a Seifert fibration with orbit surface an annulus and one singular fibre of multiplicity $q$. Let $N$ be a 0 -framed tubular neighbourhood of $C_{p, q}$ in $S^{1} \times D^{2}$. Then it is not hard to show that the ordinary fibres on $\partial N$ are $(p q, 1)$-curves on $\partial N$.

Now consider $\left(C_{p, q} ; r\right)=Y \cup V$, where $V$ is a solid torus and $\partial V$ is identified with $\partial N$. If the boundary of a meridian disc of $V(\operatorname{an}(m, n)$-curve on $\partial N)$ does not correspond to an ordinary fibre on $\partial N$, that is, if $r \neq p q$, then the Seifert fibration of $Y$ extends to one of $\left(C_{p, q} ; r\right)$. This has orbit surface $D^{2}$ and (possibly) singular fibres of multiplicities $q$ and $|n p q-m|(n p q-m$ is the intersection number of a $(p q, 1)$ curve on $\partial N$ with an $(m, n)$-curve). If $n p q-m= \pm 1$, then we have only one singular fibre, so that $\left(C_{p, q} ; r\right) \cong S^{1} \times D^{2}$. Otherwise, there are two singular fibres, and $\left(C_{p, q} ; r\right)$ is a Seifert fibre space with incompressible boundary.

It remains to consider the case $r=p q$. To do this, let $W=S^{1} \times \frac{1}{2} D^{2} \subset S^{1} \times D^{2}$, so that $C_{p, q} \subset \partial W$. Then $Y$ may be expressed as $W \cup_{A} Z$, where $Z \cong S^{1} \times S^{1} \times I$, and $A$ is an annulus which is a neighbourhood of a $(p, q)$-curve on $\partial W$, and a neighbourhood of a nontrivial curve in $S^{1} \times S^{1} \times\{0\}$. Also, $\partial A$ is a pair of $(p q, 1)$-curves on the 0 -framed tubular neighbourhood $N$ of $C_{p, q}$ in $S^{1} \times D^{2}$, separating $\partial N$ into two annuli $A_{1}, A_{2}$, say, where $A_{1} \subset \partial W$ and $A_{2} \subset \partial Z$.

Let $M=\left(C_{p, q} ; p q\right)$. Then $M \cong\left(W \cup_{A} Z\right) \cup V$, where $\partial V$ and $\partial N$ are identified in such a way that $\partial A$ bounds a pair of disjoint meridian discs $D_{1}, D_{2}$ in $V . D_{1}$ and $D_{2}$ decompose $V$ as the union of two 3-balls $B_{1}, B_{2}$, which we may suppose numbered so that the 2-sphere $A \cup D_{1} \cup D_{2}$ separates $M$ into $W \cup B_{1}$ and $Z \cup B_{2}$, where $B_{i}$ (regarded as a 2-handle) is attached along $A_{i}, i=1,2$. Thus $W \cup B_{1} \cong L(q, p)^{-}$, and $Z \cup B_{2} \cong\left(S^{1} \times D^{2}\right)^{-}$. It follows that $M \cong S^{1} \times D^{2} \# L(q, p)$, as stated. 
Since the winding number of $C_{p, q}$ is $q$, we obtain (see Lemma 3.7)

COROLlary 7.3.

$$
\left(C_{p, q}(K) ; r\right) \cong \begin{cases}(K ; p / q) \# L(q, p), & \text { if } r=p q \\ \left(K ; r / q^{2}\right), & \text { if } m=n p q \pm 1\end{cases}
$$

and otherwise, is the union (along their boundaries) of the exterior of $K$ and a Seifert fibre space with incompressible boundary.

The second case of Corollary 7.3 shows that Theorem 2(ii) of [3] is false.

Taking $K=O$ in Corollary 7.3, so that we have $C_{p, q}(O)=T_{p, q}$, the $(p, q)$-torus knot, one obtains the following. (For nontriviality, we assume here that $q \geqslant 2$ and $|p| \geqslant 2$.)

\section{COROllary 7.4 (MOSER [20]).}

$$
\left(T_{p, q} ; r\right) \cong \begin{cases}L(p, q) \# L(q, p), & \text { if } r=p q, \\ L\left(m, n q^{2}\right), & \text { if } m=n p q \pm 1,\end{cases}
$$

and otherwise is a Seifert space with orbit surface $S^{2}$ and three singular fibres of multiplicities $q,|p|$, and $|n p q-m|$.

Let $\left[p_{1}, q_{1} ; p_{2}, q_{2} ; \cdots ; p_{k}, q_{k}\right]$ denote the $\left(p_{1}, q_{1}\right)$-cable of the $\left(p_{2}, q_{2}\right)$-cable of $\cdots$ the $\left(p_{k}, q_{k}\right)$-torus knot. (As usual, we assume $q_{i} \geqslant 2,1 \leqslant i \leqslant k$, and $\left|p_{k}\right| \geqslant 2$.) Such a knot is called an iterated torus knot. Note that its exterior is a special graphmanifold (\$4).

The following theorem describes the manifolds obtained by Dehn surgery on an iterated torus knot. For brevity, we use $S$ to denote some Seifert fibre space with orbit surface $S^{2}$ and three singular fibres, and $G$ to denote some special graphmanifold with at least one incompressible torus, so that " $\cdots$ is $S$ " means “ $\cdots$ is a Seifert fibre space with orbit surface $S^{2}$ and three singular fibres", and so on. We do not specify precisely the particular $S$ 's and $G$ 's that occur, although their determining invariants could be worked out from the proof. We also assume $k \geqslant 2$, the case $k=1$ being covered by Corollary 7.4 .

THEOREM 7.5. ([ $\left.\left.p_{1}, q_{1} ; p_{2}, q_{2} ; \cdots ; p_{k}, q_{k}\right] ; r\right), r \in \mathbf{Q}$, may be described as follows:

(i) $r=p_{1} q_{1}, p_{1}=q_{1} p_{2} q_{2} \pm 1$ :

$$
\begin{array}{ll}
k=2: & L\left(p_{1}, q_{1} q_{2}^{2}\right) \# L\left(q_{1}, \pm 1\right), \\
k=3: & S \# L\left(q_{1}, \pm 1\right), \\
k>3: & G \# L\left(q_{1}, \pm 1\right) .
\end{array}
$$

(ii) $r=p_{1} q_{1}$, but not case (i):

$$
\begin{array}{ll}
k=2: & S \# L\left(q_{1}, p_{1}\right), \\
k \geqslant 3: & G \# L\left(q_{1}, p_{1}\right) .
\end{array}
$$


(iii) $q_{1}=2, p_{1}=2 p_{2} q_{2} \pm 1, r=4 p_{2} q_{2} \pm 1$ :

$$
\begin{array}{ll}
k=2: & L\left(4 p_{2} q_{2} \pm 1,4 q_{2}^{2}\right), \\
k=3: & S, \\
k>3: & G .
\end{array}
$$

(iv) $m=n p_{1} q_{1} \pm 1$, but not case (iii):

$$
\begin{array}{ll}
k=2: \quad & S, \\
k \geqslant 3: & \quad G .
\end{array}
$$

(v) otherwise:

G.

Proof. Let

$$
K=\left[p_{1}, q_{1} ; \cdots ; p_{k}, q_{k}\right], \quad K_{1}=\left[p_{2}, q_{2} ; \cdots ; p_{k}, q_{k}\right],
$$

and

$$
K_{2}=\left[p_{3}, q_{3} ; \cdots ; p_{k}, q_{k}\right], \quad \text { if } k \geqslant 3 .
$$

Then by Corollary 7.3, $M=(K ; r)=\left(C_{p_{1}, q_{1}}\left(K_{1}\right) ; r\right)$ is $G$ unless either $r=p_{1} q_{1}$ or $m=n p_{1} q_{1} \pm 1$. In particular, this establishes (v). We examine the two exceptional cases separately.

Case 1. $r=p_{1} q_{1}$. By Corollary 7.3,

$$
M \cong\left(K_{1} ; p_{1} / q_{1}\right) \# L\left(q_{1}, p_{1}\right) .
$$

If $k=2$, then $\left(K_{1} ; p_{1} / q_{1}\right)=\left(T_{p_{2}, q_{2}} ; p_{1} / q_{1}\right)$, which by Corollary $7.4\left(\right.$ since $p_{1} / q_{1}$ is not an integer), is

$$
\begin{array}{ll}
L\left(p_{1}, q_{1} q_{2}^{2}\right), & \text { if } p_{1}=q_{1} p_{2} q_{2} \pm 1, \\
S, & \text { otherwise. }
\end{array}
$$

If $k \geqslant 3$, then $\left(K_{1} ; p_{1} / q_{1}\right)=\left(C_{p_{2}, q_{2}}\left(K_{2}\right) ; p_{1} / q_{1}\right)$, and by Corollary 7.3 , this is

$$
\begin{array}{ll}
\left(K_{2} ; p_{1} / q_{1} q_{2}^{2}\right), & \text { if } p_{1}=q_{1} p_{2} q_{2} \pm 1, \\
G, & \text { otherwise. }
\end{array}
$$

In turn, by Corollaries 7.3 and $7.4,\left(K_{2} ; p_{1} / q_{1} q_{2}^{2}\right)$ is

$$
\begin{aligned}
& S, \quad \text { if } k=3, \\
& G, \quad \text { if } k>3,
\end{aligned}
$$

unless

$$
p_{1}=q_{1} q_{2}^{2} p_{3} q_{3} \pm 1 \text {. }
$$

But equations (a) and (b) imply $q_{1} q_{2}\left(p_{2}-q_{2} p_{3} q_{3}\right)=0$ or \pm 2 (according as the \pm 1 's in (a) and (b) are the same or different), both of which are impossible since $\left(p_{2}, q_{2}\right)=1$ and $q_{1}, q_{2} \geqslant 2$. Hence (b) cannot occur.

This establishes (i) and (ii).

Case 2. $m=n p_{1} q_{1} \pm 1$.

Then (by Corollary 7.3) $M \cong\left(K_{1} ; m / n q_{1}^{2}\right)$. Note that (c) implies $\left(m, q_{1}\right)=1$, so that $m / n q_{1}^{2}$ is in its lowest terms; in particular, it is not an integer.

If $k=2$, it follows from Corollary 7.4 that $M$ is

$$
\begin{array}{ll}
L\left(m, n q_{1}^{2} q_{2}^{2}\right), & \text { if } m=n q_{1}^{2} p_{2} q_{2} \pm 1, \\
S, & \text { otherwise. }
\end{array}
$$


If $k \geqslant 3$, Corollary 7.3 shows that $M$ is

$$
\begin{array}{ll}
\left(K_{2} ; m / n q_{1}^{2} q_{2}^{2}\right), & \text { if (d) holds, } \\
G, & \text { otherwise. }
\end{array}
$$

In turn, $\left(K_{2} ; m / n q_{1}^{2} q_{2}^{2}\right)$ is

$$
\begin{aligned}
& S, \quad \text { if } k=3, \\
& G, \quad \text { if } k>3,
\end{aligned}
$$

unless

$$
m=n q_{1}^{2} q_{2}^{2} p_{3} q_{3} \pm 1 \text {. }
$$

But as in Case 1 above, equations (d) and (e) are inconsistent.

It remains to examine when (c) and (d) can both hold. Subtracting, we obtain $n q_{1}\left(p_{1}-q_{1} p_{2} q_{2}\right)=0$ or \pm 2 . The first possibility gives $p_{1}=q_{1} p_{2} q_{2}$, contradicting $\left(p_{1}, q_{1}\right)=1$. So the \pm l's in (c) and (d) are different, and since $m$ and $n$ are determined only up to simultaneous sign change, we may assume that we have a -1 in (c) and $a+1$ in (d), giving

$$
n q_{1}\left(p_{1}-q_{1} p_{2} q_{2}\right)=2 \text {. }
$$

Therefore $q_{1}=2$, and $n= \pm 1, p_{1}-q_{1} p_{2} q_{2}= \pm 1$, so $p_{1}=2 p_{2} q_{2} \pm 1$. By (d), $m= \pm 4 p_{2} q_{2}+1$, giving $r=4 p_{2} q_{2} \pm 1$. Finally, if $k=2$,

$$
M \cong L\left(m, n q_{1}^{2} q_{2}^{2}\right)=L\left( \pm 4 p_{2} q_{2}+1, \pm 4 q_{2}^{2}\right) \cong L\left(4 p_{2} q_{2} \pm 1,4 q_{2}^{2}\right) .
$$

The question as to when (and which) lens spaces or connected sums of lens spaces can be obtained by Dehn surgery on an iterated torus knot is considered by Fintushel-Stern in [3]. For lens spaces, the case $k=2$ of Theorem 7.5(iii) is Theorem 1 of [3]. However, for connected sums of lens spaces, the case $k=2$ of Theorem 7.5(i) shows that the statement of Theorem 4 of [3] is incorrect. Also, in [3] the authors show that for some knot $K,(K ; \pm 155) \cong L(31,17) \# L(5,4)$, and deduce from their Theorem 4 that $K$ cannot be an iterated torus knot. However, by Theorem $7.5(i)([31,5 ; 2,3] ;-155) \cong L(31,17) \# L(5,4)$. The question whether a connected sum of nontrivial lens spaces can be obtained by Dehn surgery on a knot other than an iterated torus knot seems to still be open.

Finally, we remark that essentially the same argument as that used for the case $k>3$ of Theorem 7.5 shows the following.

THEOREM 7.6. If $K \neq O$, then $\left(C_{p_{1}, q_{1}} C_{p_{2}, q_{2}} C_{p_{3}, q_{3}}(K) ; r\right)$ contains an incompressible torus for all $r \in \mathbf{Q}$.

\section{A finiteness theorem.}

Proof OF TheOREM 1.11. We shall prove the theorem by induction on $t(K)$.

If $t(K)=0$, then $K$ is either hyperbolic or a torus knot. In the first case, $(K ; r)$ is hyperbolic (and therefore irreducible with infinite fundamental groups) for all but finitely many $r$ by [27, Theorem 5.9]. In the second case, only (i) is relevant, and this follows from Corollary 7.4.

So suppose $t(K)>0$, and consider a maximal system of tori $\mathcal{T}$ for $X$. Let $M$ be the component of $X-$ int $N(\mathcal{T})$ which contains $\partial X$. Since $\mathcal{T} \neq \varnothing, \partial M$ has at least two components. There are two cases. 
Case 1. $M$ is a Seifert fibre space. Then, by Lemma 4.1, either $K=C_{p, q}\left(K^{\prime}\right)$, $K^{\prime} \neq O$, or $K=K_{1} \# K_{2}, K_{1} \neq O \neq K_{2}$. If $K=K_{1} \# K_{2}$, the result follows from Lemma 7.1. If $K=C_{p, q}\left(K^{\prime}\right)$, then $t\left(K^{\prime}\right)<t(K)$ (by Lemma 5.2) and the result follows from Corollary 7.3 and induction.

Case 2. $M$ is hyperbolic. Let $M_{r}$ denote the result of "Dehn surgery" of type $r$ on $M$ along $\partial X$ (defined using the framing of $N(K)$ ). Then by [27, Theorem 5.9], $M_{r}$ is hyperbolic, and therefore irreducible with incompressible boundary, for all but finitely many $r$. Then, for these $r,(K ; r) \cong(\overline{X-M}) \cup M_{r}$ is irreducible and contains the (nonempty) disjoint union of incompressible tori $\partial M_{r}=\partial(\overline{X-M})$.

9. Some group-theoretic conjectures. In this section we consider the question whether $(K ; 0)$ can ever be homeomorphic to $S^{1} \times S^{2} \# M$ with $\pi_{1}(M) \neq 1$, and make some elementary remarks on some related group-theoretic questions.

Recall that a group has weight 1 if it is generated by a single conjugacy class. Since having weight 1 is preserved under passing to a quotient, and since a knot group is generated by the conjugacy class of a meridian, it follows that $\pi_{1}((K ; r))$ has weight 1 for all $r$. In particular, if $(K ; 0) \cong S^{1} \times S^{2} \# M$, then $\mathbf{Z} * \pi_{1}(M)$ has weight 1 . This raises the following question, attributed to Kervaire (see [13, pp. 116-117]).

Question 9.1. If $G \neq 1$, can $\mathbf{Z} * G$ have weight 1 ?

If follows from [4] that the answer is "no" if $G$ has a nontrivial finite quotient. (More generally, it follows from [4] that if $G$ is residually finite, and $R \in \mathbf{Z} * G$ is a relator with nonzero exponent sum in the generator of the $\mathbf{Z}$ factor, then the canonical map $G \rightarrow \mathbf{Z} * G /\langle R\rangle$ is injective.) We remark that all known (closed) 3-manifold groups satisfy this hypothesis, for the only known closed 3-manifolds are connected sums of Seifert fibre spaces, hyperbolic manifolds, and Haken manifolds. All three classes are known to have residually finite fundamental groups (the last is an unpublished result of Thurston), and residual finiteness is preserved under formation of free products [8].

In [14, Problem 5.7], Freedman notes that a nontrivial free product $G * H$ can have weight 1 if $G$ and $H$ have torsion (for example, if $G \cong \mathbf{Z}_{p}$, generated by $g$, $H \cong \mathbf{Z}_{q}$, generated by $h$, and $p, q$ are coprime, then $G * H /\langle g h\rangle=1$ ), but asks: if $G$ is torsion-free, is one of the canonical maps $G, H \rightarrow G * H /\langle R\rangle$ always an injection, for any $R \in G * H$ ? The answer to this is "no", however. For if $g \in G \neq 1$ satisfies $G /\left\langle g^{n}\right\rangle=1$, for some integer $n>1$, and $h \in H$ has order $n$ and satisfies $H /\langle h\rangle=1$, then $G * H /\langle g h\rangle=1$. It is not hard to find examples of this kind with $G$ torsion-free. Indeed, they exist in the context of Dehn surgery, for Corollary 7.3 shows that for any $K, p, q, \pi_{1}((K ; p / q)) * \mathbf{Z}_{q}$ has weight 1 , and one can certainly arrange that $\pi_{1}((K ; p / q))$ be torsion-free (for example, this will always be the case if $K$ is composite, by Lemma 7.1).

A more appropriate conjecture is perhaps the following.

Conjecture 9.2. If $G$ and $H$ are torsion-free, and $R \in G * H$, then one of the canonical maps $G, H \rightarrow G * H /\langle R\rangle$ is injective.

Closely related to this is

Conjecture 9.3. If $G$ and $H$ are nontrivial and torsion-free, and $R \in G * H$, then $G * H /\langle R\rangle$ is nontrivial. 
Certainly Conjecture 9.2 implies Conjecture 9.3, and for countable groups, they are equivalent, since any countable torsion-free group can be embedded in a countable torsion-free simple group. (For example, use the $\mathrm{HNN}$-construction to embed the given group in one such that all nontrivial elements are conjugate, as in [18, Theorem 3.3, pp. 188-189].)

Another related group-theoretic conjecture is the following.

Conjecture 9.4. If $R \in G_{1} * G_{2} * G_{3}$, then the canonical map $G_{i} \rightarrow G_{1} * G_{2} * G_{3} /\langle R\rangle$ is injective for some $i, 1 \leqslant i \leqslant 3$.

Again, since any countable group can be embedded in a countable simple group [18, pp. 188-189], for countable groups this is equivalent to

Conjecture 9.5. If $G_{i} \neq 1, i=1,2,3$, and $R \in G_{1} * G_{2} * G_{3}$, then $G_{1} * G_{2} * G_{3} /\langle R\rangle \neq 1$.

This would of course imply the impossibility of obtaining the connected sum of three nonsimply-connected manifolds by Dehn surgery on a knot. (This question is raised in [3].)

An interesting first case of Conjecture 9.5 to consider might be $\mathbf{Z}_{2} * \mathbf{Z}_{3} * \mathbf{Z}_{5}$.

Finally, we observe that the impossibility of obtaining $S^{1} \times S^{2} \# M$ with $\pi_{1}(M)$ $\neq 1$ by surgery on a knot would follow from the Whitehead conjecture that a subcomplex of an aspherical 2-complex is aspherical [29].

Let $G$ be a perfect group such that there exists a connected 2-complex $X$ with $\pi_{1}(X) \cong G, H_{2}(X)=0$, and $\pi_{2}(X) \neq 0$. Note that the fundamental group of any nonsimply-connected homology 3-sphere $M$ is such a group (take $X$ to be a 2-dimensional spine of $M^{-}$).

THEOREM 9.6. Let $G$ be as above, $F$ a free group of rank $n$, and $R_{1}, \ldots, R_{n} \in G * F$. Then the Whitehead conjecture implies that $G * F /\left\langle R_{1}, \ldots, R_{n}\right\rangle \neq 1$.

Proof. We have $G * F /\left\langle R_{1}, \ldots, R_{n}\right\rangle \cong \pi_{1}(Y)$, where $Y$ is the 2-complex obtained from $X$ by adding $n$ 1-cells and $n$ 2-cells in the obvious way. Since $H_{1}(X)=H_{2}(X)$ $=0$, the euler characteristic $\chi(X)=1$. Hence $\chi(Y)=1$. Now suppose $\pi_{1}(Y)=1$. Then $H_{2}(Y)=0$, and hence $Y$ is contractible. The Whitehead conjecture would then imply $\pi_{2}(X)=0$, a contradiction.

COROLlaRY 9.7. The Whitehead conjecture implies that no nonsimply-connected homology 3-sphere can bound a contractible 4-manifold with no 1-handles.

COROLlARY 9.8. The Whitehead conjecture implies that $(K ; 0)$ is never homeomorphic to $S^{1} \times S^{2} \# M$ with $\pi_{1}(M) \neq 1$.

10. Superslice knots. Adopting the terminology of Brakes [2], we say that a knot $K$ is superslice if $K$ bounds a disc $D$ in $B^{4}$ such that the double $2\left(B^{4}, D\right)$ is homeomorphic to the standard (unknotted) sphere pair $\left(S^{4}, S^{2}\right)$. Equivalently (by [24]), $\left(B^{4}, D\right) \times I$ is homeomorphic to the standard pair $\left(B^{5}, B^{3}\right)$; see [7] for examples. Let us also say that $K$ is homotopy-superslice if $\left(S^{3}, K\right)=\partial(V, D)$ for some (locally flat) disc $D$ in a homotopy 4-ball $V$, such that $2(V, D) \cong(\Sigma, S)$, say, where $\Sigma-S$ is a homotopy $S^{1}$. (By a fairly straightforward duality argument in the infinite cyclic covering, $\Sigma-S$ is a homotopy $S^{1}$ if and only if $\pi_{1}(\Sigma-S) \cong \mathbf{Z}$.) 
Kirby-Melvin [15] show that $(K ; 0) \cong S^{1} \times S^{2}$ implies that $K$ is superslice. Related to this, one has the following, which is presumably well known to the experts.

THEOREM 10.1. If $(K ; 0) \cong S^{1} \times S^{1} \# M$, then

(i) $M$ is the boundary of a contractible 4-manifold with one 0-handle, no 1-handles, one 2-handle, and one 3-handle;

(ii) $K$ is homotopy-superslice.

Proof. (i) Let $B^{4} \cup h^{2}$ be the result of adding a 2-handle $h^{2}$ to $B^{4}$ along $K$ with the 0 -framing, so that we have $\partial\left(B^{4} \cup h^{2}\right) \cong(K ; 0) \cong S^{1} \times S^{2} \# M$. Now let $h^{3}$ be a 3-handle whose attaching sphere is $* \times S^{2} \subset\left(S^{1} \times S^{2}\right)^{-} \subset S^{1} \times S^{2} \# M$. Let $W=B^{4} \cup h^{2} \cup h^{3}$. Then $\partial W \cong M$, and $W$, being simply-connected and acyclic, is contractible.

(ii) Consider the double $2 W$ of $W$, and denote the two copies of $W$ in $2 W$ by $W_{1}$ and $W_{2}$. Let $B$ be the copy of $B^{4}$ in $W_{1}$, say, and let $V=2 W-$ int $B$. Then $V$ is a homotopy 4-ball, and $K \subset \partial V$ bounds the 2-disc $D \subset W_{1}-$ int $B \subset V$, where $D$ is the core of the 2-handle $h^{2}$ in $W_{1}$. Note also that $V-D \simeq V-h^{2} \cong W_{2} \cup h^{1}$, where $h^{1}$ is the dual 1-handle to $h^{3}$ in $W_{1}$. Since $W_{2}$ is contractible, it follows that $V-D \simeq S^{1}$. Now let $(\Sigma, S)=2(V, D)$. Then $\Sigma-S=2(V-D)$, so that $\pi_{1}(\Sigma-S) \cong \mathbf{Z}$, and hence $\Sigma-S \simeq S^{1}$.

To show that Theorem 1.5 does not follow from superslice considerations, for example, Theorem 10.1(ii), we observe that there exist satellites with arbitrary winding number which are superslice. This follows (for example with $J(O)=O$ ) from

THEOREM 10.2. If $K$ and $J(O)$ are superslice, then $J(K)$ is superslice.

Proof. Let $\left(S^{3}, K\right)=\partial\left(B^{4}, D\right)$, where $2\left(B^{4}, D\right) \cong\left(S^{4}, S^{2}\right)$. Let $g: D^{2} \times \frac{1}{2} D^{2} \rightarrow$ $B^{4}$ be an embedding onto a tubular neighbourhood of $D=g\left(D^{2} \times(0,0)\right)$. It is convenient to assume (as we may do, by an isotopy) that $J \subset S^{1} \times \frac{1}{2} D^{2}$. Since $J(O)$ is superslice, $J$ bounds a disc $\Delta \subset D^{2} \times \frac{1}{2} D^{2} \subset D^{2} \times D^{2}$ such that $2\left(D^{2} \times D^{2}, \Delta\right)$ $\cong\left(S^{4}, S^{2}\right)$. Then $J(K)=g(J)$ bounds the disc $g(\Delta) \subset B^{4}$.

In the double $2\left(D^{2} \times D^{2}\right)$, let the two copies of the first $D^{2}$-factor be $D_{+}, D_{-}$. Then $D_{+} \times \frac{1}{2} D^{2}$ and $D_{-} \times \frac{1}{2} D^{2}$ are identified along $\partial D_{ \pm} \times \frac{1}{2} D^{2}$, and the closure of the complement of $\left(D_{+} \cup D_{-}\right) \times \frac{1}{2} D^{2}$ in $2\left(D^{2} \times D^{2}\right)$ is homeomorphic to $D^{3} \times S^{1}$.

Let $\bar{g}:\left(D_{+} \cup D_{-}\right) \times \frac{1}{2} D^{2} \rightarrow 2 B^{4} \cong S^{4}$ be the embedding determined by $g$ in the obvious way. Since $2 D$ is unknotted in $S^{4}$, the closure of the complement of the image of $\bar{g}$ in $S^{4}$ is homeomorphic to $D^{3} \times S^{1}$. Since any homeomorphism of $S^{2} \times S^{1}$ extends over $D^{3} \times S^{1}$, it follows that $\bar{g}$ extends to a homeomorphism $2\left(D^{2} \times D^{2}\right) \rightarrow S^{4}$. Thus $2 g(\Delta)=\bar{g}(2 \Delta)$ is unknotted in $S^{4}$, showing that $J(K)$ is superslice.

For comparison with Theorem 10.2, we take this opportunity to remark that the proof given in $[7, \mathrm{III}]$ actually proves a little more than is explicitly stated there (in Theorem 3.1), namely 
THEOREM 10.3. If $w=0, K$ is slice and $J(O)$ is superslice, then $J(K)$ is superslice.

([7, Theorem 3.1] has the stronger hypothesis $J(O)=O$.)

\section{REFERENCES}

I. R. H. Bing and J. M. Martin, Cubes with knotted holes, Trans. Amer. Math. Soc. 155 (1971), 217-331.

2. W. R. Brakes, Property R and superslices, Quart. J. Math. Oxford 31 (1980), 263-281.

3. R. Fintushel and R. J. Stern, Constructing lens spaces by surgery on knots, Math. Z. 175 (1980), 33-51.

4. M. Gerstenhaber and O. S. Rothaus, The solution of sets of equations in groups, Proc. Nat. Acad. Sci. U.S.A. 48 (1962), 1531-1533.

5. F. González-Acuña, Dehn's construction on knots, Bol. Soc. Mat. Mexicana 15 (1970), 58-79.

6. C. McA. Gordon, Knots, homology spheres, and contractible 4-manifolds, Topology 14 (1975), $151-172$.

7. C. McA. Gordon and D. W. Sumners, Knotted ball pairs whose product with an interval is unknotted, Math. Ann. 217 (1975), 47-52.

8. K. W. Gruenberg, Residual properties of infinite soluble groups, Proc. London Math. Soc. 7 (1957), $29-62$.

9. A. Hatcher, On the boundary curves of incompressible surfaces (to appear).

10. J. Hempel, 3-Manifolds, Ann. of Math. Studies, No. 86, Princeton Univ. Press, Princeton, N.J., 1976.

11. W. H. Jaco and P. B. Shalen, Seifert fibred spaces in 3-manifolds, Mem. Amer. Math. Soc. 21 (1979).

12. K. Johannson, Homotopy equivalences of 3-manifolds with boundaries, Lecture Notes in Math., vol. 761, Springer-Verlag, Berlin, Heidelberg and New York, 1979.

13. M. A. Kervaire, On higher dimensional knots, Differential and Combinatorial Topology, A Symposium in Honor of Marston Morse (S. S. Cairns, Editor), Princeton Univ. Press, Princeton, N.J., 1965, pp. 105-119.

14. R. Kirby, Problems in low dimensional manifold theory, Proc. Amer. Math. Soc. Summer Institute in Topology, Stanford, (1976), Amer. Math. Soc., Providence, R.I., Proc. Sympos. Pure Math., Vol. 32, 1978, pp. 273-312.

15. R. Kirby and P. Melvin, Slice knots and Property R, Invent. Math. 45 (1978), 57-59.

16. R. A. Litherland, Surgery on knots in solid tori, Proc. London Math. Soc. 39 (1979), 130-146.

17. __ Surgery on knots in solid tori. II, J. London Math. Soc. 22 (1980), 559-569.

18. R. C. Lyndon and P. E. Schupp, Combinatorial group theory, Ergebnisse der Math. und ihrer Grenzgebiete, Band 89, Springer-Verlag, Berlin, Heidelberg and New York, 1977.

19. J. W. Morgan, Morse-Smale flows on 3-dimensional manifolds, Topology 18 (1979), 41-53.

20. L. Moser, Elementary surgery along a torus knot, Pacific J. Math. 38 (1971), 737-745.

21. D. Rolfsen, Knots and links, Mathematics Lecture Series, No. 7, Publish or Perish, Berkeley, Calif., 1976.

22. H. Schubert, Knoten und Vollringe, Acta Math. 90 (1953), 131-286.

23. H. Seifert, Topologie dreidimensionaler gefaserter Räume, Acta. Math. 60 (1933), 147-238.

24. J. L. Shaneson, Embeddings with codimension two of spheres in spheres and H-cobordisms of $S^{1} \times S^{3}$, Bull. Amer. Math. Soc. 74 (1968), 972-974.

25. L. Siebenmann, On vanishing of the Rohlin invariant and nonfinitely amphicheiral homology 3-spheres, Proc. Topology Sympos., Siegen, 1979 (U. Koschorke and W. D. Neumann, Editors), Lecture Notes in Math., vol. 788, Springer-Verlag, Berlin, Heidelberg and New York, 1980.

26. W. Thurston, On the geometry and dynamics of diffeomorphisms of surfaces. I (to appear).

27. ,The geometry and topology of 3-manifolds, preprints, Princeton University.

28. F. Waldhausen, Eine Klasse von 3-dimensionalen Mannigfaltigkeiten. I, II, Invent. Math. 3 (1967), 308-333; 4 (1967), 87-117.

29. J. H. C. Whitehead, On adding relations to homotopy groups, Ann. of Math. (2) 42 (1941), 409-428.

Department of Mathematics, University of Texas, Austin, Texas 78712 